Reaction Kinetics and Catalysis Letters, Vol. 1, No. 1, 57-66 /1974/

\title{
A NOTE ON A STATISTICAL-MECHANICAL TREATMENT OF ACTIVATION-LIMTT ED SURFACE DIFFUSION
}

\author{
J.D. Goddard and G. Parravano \\ University of Michigan \\ Ann Arbor, Michigan 48104, USA \\ Department of Chemical Engineering
}

Received: July 16,1973

\begin{abstract}
A brief review is given of a class of simple statistical-mechanical models for surface diffusion, applicable to the limiting case where diffusional "hopping" is controlled by the thermal activation rate of the adsorbed particle. A theoretical result of Reyes, for the pre-exponential or "frequency" factor, is discussed and compared briefly to experiment.

Привопится краткий обвор класса простых статистическо-

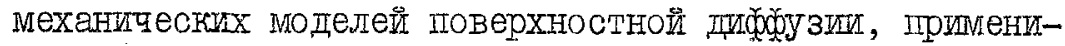
мых в предельных случалх, копда писыбузионные "прыжги" контролируются скоростью термшческой активапии здсорбированных части. Теоретические результаты Рейса относительно предәкспоненцальното множителя или "частотного" фактора обсуждаются и сравниватотся с экспердментальными значениям.
\end{abstract}

\section{INTRODUCTION}

The translational diffusion of physically adsorbed species on solid surfaces is known to play an important role in the kinetics of surface processes such as sintering or crystal growth and, perhaps, as well in the rates of some solid catalyzed reactions $[1,2]$. It is generally believed that the predominant mechanism for surface diffusion is Brownian ("random-walk") motion of the adsorbed species, sustained by thermo-molecular agitation or vibration of the substrate $[1,2,3]$.

The estimation of diffusion rates, by means of the classical microscopic description, requires a knowledge of the relevant diffusion coefficients, which in the simplest case of a (macroscopically) isotropic sarface, reduce to a single diffusivity, 
D, (area/time), say. Measured surface diffusivities usually exhibit the "Arrhenius" temperature dependence typical of "activated" transport phenomena [3], such as bulk diffusion in condensed phases, i.e.

$$
\mathrm{D}=\mathrm{D}_{\mathrm{o}} \exp \{-\mathrm{E} / \mathrm{RT}\}
$$

where the activation energy $E$ and pre-exponential factor $D_{0}$ are independent of, or weakly dependent on, temperature $T$.

The earliest theoretical descriptions $[2,3]$ of this phenomena are to be found in the works of Lennard-Jones $[4,5]$ and of Taylor and Langmuir [6], who portray it as a random walk of the particle over the potential-energy surface associated with the substrate. In the "activation-limited" case, where the random-walk is limited by the rate of activation leading to escape from a "potential well", one has in the classical way $[3,5]$

$$
\mathrm{D}=\frac{1}{4} \ell^{2} \gamma
$$

where $\gamma$ (time $e^{-1}$ ) is the mean frequency of escape and $\ell^{2}$ is the mean-square displacement or step-size. As a first approximation, $l^{2}$ can presumably be treated as a constant and equated with some characteristic surface (or "lattice") dimension, such as the mean-square separation of the potential wells, whereas the unknown quantity of primary importance is the frequency or rate of escape $\gamma$. Consistent with (1), the latter can be expressed as

$$
\gamma=\gamma_{0} \exp (-E / R T)
$$

such that the pre-exponential factor in (1) becomes

$$
D_{0}=\frac{l^{2} \gamma_{0}}{4}
$$

In the simple model, the correlation or prediction of surface diffusivity rests mainly on the possibility of relating $E, l^{2}$, and $\gamma_{0}$ to the molecular properties of the substrate and the diffusing species.

Equation (3), like (1), has a form predicted by the classical "transition-state" theory, and given an appropriate potential-energy surface to describe the intermolec- 
ular forces between substrate and adsorbed particle, one can in principle assign appropriate values of the activation "barrier height" $E$ (in addition to the mean-square potential-well separation distance $\ell^{2}$ ).

However, even if it were possible to estimate $\mathrm{E}$ (and $\ell^{3}$ ) with sufficient accuracy, which generally is not, there still remains the problem of relating the frequency factor $\gamma_{0}$ in (2) to molecular properties. Owing to its well known limitations, transition-state theory [3] does not provide generally reliable estimates of $\gamma_{0}$, and one must turn to a somewhat more detailed, if approximate statistical-mechanical theory, which is the main subject of this note.

In particular, we should like to report here on some heretofore unpublished progress [7] on a model for Brownian motion of a particle coupled to a "crystalline') lattice, which starts from equation (2), and employs the statistical mechanics of weakly-coupled systems to derive $\gamma$, the frequency of escape over a potential barrier.

The earliest such treatment is that of Lennard-Jones and Strachan [8], who derived an expression for the phonon-induced quantum-mechanical transition probability (or frequency) between the discrete energy levels of a particle in a potential "well" or "valley" on a two-dimensional crystalline surface, a treatment which is limited to single-phonon excitations. In a later work, Reyes [7] has treated classical version of this model, using the methods for weakly-coupled (classical) systems, developed by the Kirkwood [9] and Prigogine [10, 11] Schools.

As indicated in the early work of Lennard-Jones and Strachan [8], this type of theory does not really describe Brownian motion in the spatial sense but, rather, the motion of a particle amongst the energy levels in a potential well, leading ultimately to escape. As such, the theory is of same type as that proposed by Christensen $[12]$, and elaborated on by Bak and co-workers $[13,14]$ to describe the kinetics of chemical reaction, wherein "annihilation" or escape over a potential barrier is taken as the criterion for reaction.

At the outset, then, it can be anticipated that the model can provide only an approximate description of spatial transport, which, inter alia, does no take proper account of the complete geometry of potential energy surfaces and the associated particle trajectories. Nevertheless, it might be hoped to provide useful approximate results or to suggest more defined treatments. 
J. D. GODDARD, G. PARRA VANO: TREA TMENT OF ACTIVATION-LIMITED SURFACE DIFFUSION

\section{(CLASSICAL) BROWNIAN MOTION IN ENERGY SPACE OF A PARTICLE COUPLED TO A LATTICE}

To date, the only reasonably complete (classical) statistical-mechanical treatments of a particle coupled to a lattice in thermal equilibrium, are those given by Bak et al. [15] and also by Prigogine and co-workers [11, 13] for "weakly anharmonic" coupling. Their derivations, based on the time-dependent perturbation of the Liouville equation developed by Brout and Prigogine and co-workers $[10,11]$, lead to a particle "diffusion" equation in energy space of the form

$$
\frac{\partial \varphi}{\partial \tau}=\frac{\partial}{\partial \varepsilon} \varepsilon\left(1+\frac{\partial}{\partial \varepsilon}\right) \varphi
$$

where $\varphi(\varepsilon, \tau)$ is the energy distribution function of the particle, and $\varepsilon, \tau$ are the dimensionless forms of the particle energy $E_{p}$ and the time $t$ :

$$
\begin{aligned}
\varepsilon & =\mathrm{E}_{\mathrm{p}} / \mathrm{kT} \\
\tau & =\mathrm{t} / \mathrm{t} r
\end{aligned}
$$

Here, $t_{r}$ is a characteristic ("relaxation") time which in principle can be derived from. the (harmonic) vibrational frequency spectrum of particle and lattice and the coefficients of anharmonicity (i.e., the third-order derivatives of potential energy with respect to displacements from equilibrium of particle and lattice), by formulae which are given by Prigogine [11] and which shall not trouble to repeat here, We note, however, that with the assumption of a purely harmonic lattice, the approximation of a Debye spectrum of vibrational frequencies, and other approximations of the type used in the quantum theory of solids, Bak et al. [15] offer an explicit and simple expression for the characteristic time $t_{r}$.

It should be pointed out that all the above-mentioned derivations of equation (4) are based on a one-dimensional model involving an infinite linear lattice, having the associated normal harmonic vibrational modes, and an essential one-dimensional particle-lattice interaction. Since, however, equation (4) is specialized to the energy distribution this limitation is perhaps not as severe as certain others. At any rate, a more correct equation for a three-dimensional model could probably be derived in a similar way, should the basic success of the one-dimensional theory warrant it. 
As pointed out by Bak et al. $[13,14]$ and also by Prigogine (pp. 75ff.) [11], equation (4) has precisely the form given by the phenomenological theory of Brownian Motion elaborated on by Kramers [16], if the particle "friction coefficient" (force per unit momentum) is taken to be

$$
\eta=\frac{1}{t_{r}}
$$

As further pointed out by Bak and Andersen [13], this offers limited support for Kramers' theory, which they propose to account approximately for the effects of strong anharmonicity of particle binding. They thus obtain an appropriately modified form of (4) involving an energy-dependent frequency $\omega(\varepsilon)$ to account for the non-linear vibrational behaviour of the particle.

At any rate, given an exact or an approximate equation, such as $(4)$, to describe the time evolution of the particle energy distribution $\varphi(\varepsilon, \tau)$ one is then in a position to estimate the rate of "annihilation" or escape of particles over a potential barrier. Thus, following Bak and co-workers $[13,14]$, one takes, for a barrier height $E$, the following asymptotic form for the frequency $\gamma$ in (2),

$$
\gamma=-\operatorname{Lim}_{t \rightarrow \infty} \frac{d}{d t}\left\{\ln \left[\int_{0}^{\varepsilon^{*}} \varphi(\varepsilon, \tau) d \varepsilon\right]\right\}
$$

where $\varphi$ is a solution to the evolution equation, e.g. (4), subject to an arbitrary initial condition at $t=0$ in $\left(0, \varepsilon^{*}\right)$ and a boundary condition

$$
\varphi\left(\varepsilon^{*}, t\right)=0
$$

at the "top" of the barrier, where $E_{p}=E$ and

$$
\varepsilon=\varepsilon^{*}=\mathrm{E} / \mathrm{kT} \text {. }
$$

In the cass of equation (4), Bak et al. [13] show that the solution for $\varphi$ reduces, by separation of variables, to a Sturm-Liouville system involving the confluent hypergeometric equation, on the interval $\left(0, \varepsilon^{*}\right)$. The corresponding eigen-value problem determines a discrete spectrum of "relaxation" times depending on $\varepsilon^{*}$, the largest of which determines the limit in (7). In this way, one finds that 
J. D. GODDARD, G. PARRAVANO: TREATMENT OF ACTIVATION-LIMITED SURFACE DIFFUSION

$$
\gamma=f\left(\varepsilon^{*}\right) \frac{\varepsilon^{*} e^{-\varepsilon^{*}}}{t_{r}}
$$

where $t_{\mathbf{r}}$ is the characteristic time of equation (4) and $f\left(\varepsilon^{*}\right)$ is a factor accounting for finite barrier height (i.e., for the boundary condition in (8) with $\varepsilon^{*}<\infty$ ), such that

$$
f\left(\varepsilon^{*}\right) \rightarrow 1 \text { for } \varepsilon^{*} \rightarrow \infty
$$

A plot of $f\left(\varepsilon^{*}\right)$ has been given by Reyes $[7]$, and for $\varepsilon^{*}=E / k T \geq 3$ one may take $f \doteq 1$, with less than $15 \%$ error.

Thus, by means of (10), the frequency $\gamma$ is seen to have a form consistent with the temperature dependence of equation (3), and on setting the factor $f$ equal to unity in (10), one has, by equations (2) and (10), the desired result for surface diffusivity:

$$
\mathrm{D}=\frac{\ell^{2}}{4 \mathrm{t}_{r}} \quad\left(\frac{\mathrm{E}}{\mathrm{kT}}\right) \exp (-\mathrm{E} / \mathrm{kT}),
$$

and hence, for the pre-exponential factor in (1):

$$
D_{0}=\frac{l^{2}}{4 t_{x}} \quad t-\frac{E}{k T}-
$$

which are the formulae proposed by Reyes [7]

\section{ESTIMATION OF MODEL PARAMETERS AND APPLICATION TO SPECIFIC PHYSICAL SYSTEMS}

In the form (12), one sees that the value of the pre-exponential factor depends on the details of the (anharmonic) lattice-particle potential energy through the characteristic time constant $t_{r}$. As mentioned above, Prigogine [11] has proposed a general expression for this quantity, while Bak et al. [15] have proposed a greatly simplified form based on a purely harmonic lattice and certain other approximations, whose exact validity is somewhat difficult to assess.

On the other hand, Reyes [7] has derived a fairly explicit expression in terms of lattice parameters. In his treatment, which follows closely that of Bak et al. [15], he assumes a purely harmonic lattice with only nearest neighbor interactions between 
lattice points, whereas the adsorbed particle is assumed bound to a particular lattice point, a hypothetical "surface" atom*, by a Morse potential,

$$
U(r)=U_{\infty}\left(1-e^{-\frac{r}{\sigma}}\right)^{2}
$$

where $U_{\infty}$ is the dissociation energy, $\sigma$ the characteristic length scale, and $r$ the displacement from the equilibrium separation of particle and lattice point. Ultimately, however, in the work of Reyes [7] this potential is effectively replaced by the simplified, small-r expansion

$$
\begin{aligned}
\mathrm{U}(\mathrm{r}) & =\mathrm{U}_{\infty}\left(\frac{\mathrm{r}}{\sigma}\right)^{2}\left(1+\frac{\mathrm{r}}{\sigma}\right), \\
& =\frac{\mathrm{m \omega _{0 }}{ }^{2} \mathrm{r}^{2}}{2}\left(1+\frac{\mathrm{r}}{\sigma}\right)
\end{aligned}
$$

Here,

$$
\omega_{0}^{2}=\frac{2}{m}\left(\frac{U_{\infty}}{\sigma^{2}}\right)
$$

is the associated harmonic (or low-energy) frequency of the "unperturbed" adsorbed particle, with $\mathrm{m}$ denoting the particle mass. Equation (13) corresponds to the usual anharmonic approximation for the lattice-particle coupling terms, while accounting for a weak non-linearity in the "unperturbed" particle vibration.

On replacing the resultant energy-dependent $\omega(\varepsilon)$ particle frequency by an effective, constant average frequency $\bar{\omega}$ Reyes [7] obtains the following result for the time constant $t_{r}$ in (12):

$$
t_{r}=\frac{1}{2 \omega_{o}} \frac{m_{c} \omega_{c}}{m \omega_{o}} \quad \sqrt{1-\left(\frac{\bar{\omega}}{\omega_{c}}\right)^{2}}
$$

\footnotetext{
*While Reyes [7] purports to treat the special lattice point as a "surface atom", he in fact employs the vibrational modes for an infinite lattice without edge effects and chooses to regard this as the "neglect of the surface mode on the lattice". However one does this, it amounts to making no distinction between the infinite and semi-infinite linear lattices. (cf. Lennard-Jones and Strachan [8], who make the same type of approximation.)
} 
where $m_{c}$ is the mass of a lattice particle and $\omega_{c}$ is the maximum lattice vibrational frequency, related [7] to the speed of sound $c$ and the interatomic spacing in the lattice d by

$$
\omega_{c}=\frac{2 c}{d}
$$

By then using the somewhat arbitrary value

$$
\bar{\omega}=\omega_{0}\left[1-\frac{E}{4 U_{\infty}}\right]
$$

in (15) and by further equating the diffusion step size $l$ in (12) to the lattice parameter $d$, Reyes [7] obtains an expression for the pre-exponential factor $D_{0}$, in terms of quantities that can be estimated for several systems that have been studied experimentally. He has performed calculations of the pre-exponential factor $D$ for some 17 systems, for which diffusion data were available and estimates of the other parameters could be made.

Without repeating all the results here, we note simply the two extremes of agreement of the theoretical formula with experiment which, as it turns out, both involve self diffusion:

For the system Ni on Ni (diffusion data from Ref. [17], random surface crystalline orientation, temperature range $1073-1473 \mathrm{~K}$, observed $\mathrm{E}=39.2 \mathrm{kcal} / \mathrm{mol}$, calculation made for $\mathrm{E} / \mathrm{RT}=0.4$ ) the observed $\mathrm{D}_{\mathrm{o}}=2.1 \times 10^{-2} \mathrm{~cm}^{2} / \mathrm{s}$ and the calculated $\mathrm{D}_{\mathrm{o}}=3.57 \times 10^{-2} \mathrm{~cm}^{2} / \mathrm{s}$, which must be regarded as very good.

On the other hand, for the system $\mathrm{Fe}$ on $\mathrm{Fe}$ (diffusion data from Ref. [18], random surface crystalline orientation, temperature range 1203-1308 $\mathrm{K}$, observed $\mathrm{E}=40 \mathrm{kcal} / \mathrm{mol}$, calculation made for $\mathrm{E} / \mathrm{RT}=16.0$ ) the observed $\mathrm{D}_{\mathrm{o}}=5.4 \times 10^{5} \mathrm{~cm}^{2} / \mathrm{s}$ and the calculated $\mathrm{D}_{\mathrm{o}}=1.15 \times 10^{-1} \mathrm{~cm}^{2} / \mathrm{s}$, which corresponds to $\mathrm{D}_{\mathrm{o}}$ (calc.) $/ \mathrm{D}_{\mathrm{o}}(\mathrm{Exp})=$ $=2.13 \times 10^{-7}$.

Other calculations, some for foreign metallic atoms on metallic substrates, range between these extremes, the closest agreement (within one to two orders of magnitude) occurring for experimental values of $\mathrm{D}_{\mathrm{o}}$ on the order of $10^{-1}$ to $10^{-3} \mathrm{~cm}^{2} / \mathrm{s}$, and the worst agreement corresponding to the larger experimental $D_{0}$ values, on the order of 1 to $10^{5} \mathrm{~cm}^{2} / \mathrm{s}$. 
There are, of coilrse, numerous plausible reasons for the lack of success of the above simple model. It is somewhat doubtful that the exact form of the one-dimensional particle-lattice potential is important, since Bak and Andersen [13] have shown that more complicated forms of the energy-distribution equation, based on Kramers' phenomenological theory of Brownian motion with strongly anharmonic binding of the diffusing particle, lead to essentially the same orders of magnitude of dimensionless escape rates $\gamma \mathrm{t}_{\mathbf{r}}$ over a potential barrier.

More plausibly, it would seem that the type of "activation-limited" model discussed here does not provide an adequate model of spatial transport. Hence, as already anticipated in the very earliest works of Lennard-Jones [5] and Langmuir [6], it will probably be necessary to develop statistical-mechanical models which account properly for "time of flight", or mean-square displacement $\ell^{2}$, by means of an appropriate description of particle-lattice potential energy surfaces and translational momentum exchange. In this respect, a theory of spatial transport, akin to the "highenergy" particle limit considered by Bak et al. [15], would be desirable.

\section{ACKNOWLEDGEMENT}

This work draws heavily on the unpublished Ph.D. Dissertation of Dr. R. Reyes [7], copies of which may be obtained from University Microfilms.

\section{REFERENCES}

[1]. N.A.Gjostein, in "Surfaces and Interfaces, I. Chemical and Physical Characteristics," (Burke et al. eds.) Syracuse Iniversity Press, 1967.

[2]. H.P. Bonzel, : "Surface Diffusion of Metals," Scientific Research Staff, Publication Preprint, Ford Motor Co., Research Laboratories, Metallurgy Department, Dearborn, Michigan, (February 1) 1972.

[3] J.M. Blakely: Prog. Materials Sci., 10, 395 (1963)

[4]. L.E. Lennard-Jones: Trans. Faraday Soc., 28, 33 (1932)

[5]. L. E. Lennard-Jones: Proc. Phys. Soc. (London), 49, 140 (1937)

[6]. J.B. Taylor and I. Langmuir: Phys. Rev., 44, 423 (1933)

[7]. R.R. Reyes: "A Non-Equilibrium Statistical Mechanical Model For Surface Diffusion," Ph.D. Dissertation, University of Michigan, Ann Arbor, 1966.

[8.] L. E. Lennard-Jones and C.Strachan: Proc. Roy. Soc. (London), A 150, 442, (1935) 
[9]. J.G. Kirkwood: "Selected Topics in Statistical Mechanics," Chapter I ffrom J. Chem. Phys., 14, 180 (1946), Gordon and Breach, New York 1967.

[10]. R. Brout and I. Prigogine: Physica, 22, 621 (1956)

[11]. I. Prigogine: "Non-Equilibrium Statistical Mechanics," Interscience Publ., New iork, 1962.

[12]. J.A. Christensen: J. Phys. Chem., 37, 374 (1937)

[13]. T.A. Bak and D. Andersen: Mat. Phys. Medd. Dan. Vid. Selsk., 33, No. 7 (1961)

[14]. T.A.Bak and E.R. Fisher: "Applied Kinetics," Ch. 7, pp. 187 ff., Am. Chem. Soc. Publications, Washington D.C., 1967.

[15]. T.A. Bak, M. Goche and F.Henin: J. Mol. Phys., 22 181 (1959)

[16] H. A. Kramers: Physica, 7, 284 (1940)

[17]. J.M. Blakely: and H. Mykura: Acta, Met., 9, 23 (1961)

[18]. J. M. Blakely and H. Mykura: Acta. Met., 10, 565 (1962) 